\title{
Unmasking normocalcaemic hyperparathyroidism (nPHPT) resulting in severe hypercalcaemia from lack of monitoring during in the COVID pandemic
}

\begin{abstract}
Primary hyperparathyroidism (PHPT) is a common endocrine disorder in the older person although the incidence of normocalcaemic primary hyperparathyroidism (nPHPT) remains largely unknown. Whilst the majority of patients with PHPT asymptomatic, we describe a case of severe symptomatic hypercalcaemia from undiagnosed nPHPT whilst on calcium and vitamin D supplements. This is an important reminder to clinicians that serum calcium should be monitored before and after initiation of calcium and vitamin D supplementation in the older person.
\end{abstract}

Keywords: primary hyperparathyroidism, normocalcaemic primary hyperparathyroidism ,calcium and vitamin d supplements, older people
Volume 6 Issue 2 - 2021

\author{
Peter Chua,' Su-Lee Xiao, ${ }^{2}$ Elaine Hui, ${ }^{3}$ \\ Yetunde Baoku, ${ }^{4}$ Eddy Chua $^{2}$ \\ 'University of Manchester, Faculty of Medicine, UK \\ ${ }^{2}$ Department of Medicine for the Older Person, Northwick \\ Park Hospital, UK \\ ${ }^{3}$ Department of Endocrinology, Northwick Park Hospital, UK \\ ${ }^{4}$ Clinical Biochemistry, Northwick Park Hospital, UK
}

Correspondence: Dr Eddy Chua, Department of Medicine for the Older Person, Northwick Park Hospital,Watford Road, Harrow, HAI 3UJ, Email eddy.chua@nhs.net

Received: March 23, 202I | Published: April 08, 202।

\section{Key points}

Primary hyperparathyroidism is common in older people, and the majority are asymptomatic.

Raised calcium with a normal parathyroid hormone does not exclude primary hyperparathyroidism.

Serum parathyroid hormone is not routinely checked before initiating calcium and vitamin $\mathrm{D}$ in the older person.

It is important to check serum calcium before and after initiation of calcium and vitamin D supplements.

\section{Case report}

A 75-year-old lady presented with acute hypoactive delirium. She had a history of osteoporosis, falls, neck of femur fracture, breast cancer and chronic obstructive pulmonary disease. Her medications included calcium and vitamin D for secondary fracture prevention. She had annual blood test via her general practitioner. Herrenal function serum calcium and vitamin $\mathrm{D}$ had been normal over the past two consecutive years, however she had missed her test in April 2020 due to COVID-19.

On admission she had an abbreviated mental test score (AMTS) of 4/10. Blood tests showed creatinine 325umol/L (44-80 umol/1), urea $16.1 \mathrm{mmol} / 1$ (2.5 -7.8 mmol/1), corrected calcium of $4.87 \mathrm{mmol} / \mathrm{L}$ (normal range 2.20 - $2.60 \mathrm{mmol} / \mathrm{L}$ ), haemoglobin $112 \mathrm{~g} / \mathrm{L}$, WCC 13.6 x $10^{9} / \mathrm{L}$, neutrophils $11.0 \times 10^{9} / \mathrm{L}$, CRP $57 \mathrm{mg} / \mathrm{L}$. Sodium, potassium, and albumin were normal. Chest X-ray showed old rib fractures. Respiratory viral screen and COVID-19 swabs were negative. Renal ultra sound demonstrated no hydronephrosis. On the first day of admission, parathyroid hormone (PTH) was non-suppressed at 7.0 $\mathrm{pmol} / \mathrm{L}$ (normal range 1.6-6.9pmol/L). Following rehydration and bisphosphonate therapy, she developed a transient, asymptomatic hypocalcaemia and her PTH was elevated at $18.3 \mathrm{pmol} / \mathrm{Lon}$ the $13^{\text {th }}$ day of admission.

Investigations for hypercalcaemia excluded myeloma, sarcoidosis and hyperthyroidism. Her vitamin D was within the normal range. Her 24 hour urinary calcium: creatinine ratio was 0.021 . Contrast CT of chest, abdomen and pelvis showed norecurrence of her previous malignancy.

Calcium and vitamin D supplements were stopped. She was treated with slow intravenous fluids, and subsequently intravenous pamidronate. She also received antibiotics to cover potential infective exacerbation of COPD. Over the course of her admission, her mental state improved and her AMTS was 10 out of 10. Herrenal function and serum calcium also improved prior to hospital discharge (Figure 1). At four months post discharge, the patient has remained well with a normal serum calcium of $2.49 \mathrm{mmol} / \mathrm{L}$ and an elevated PTH $16.6 \mathrm{pmol} / \mathrm{L}$.

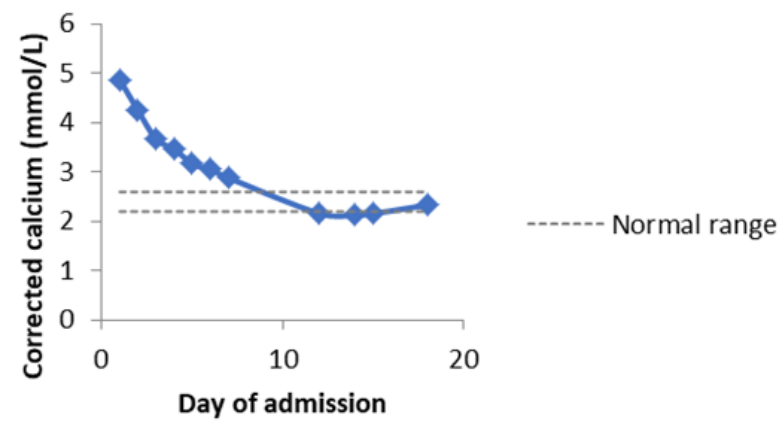




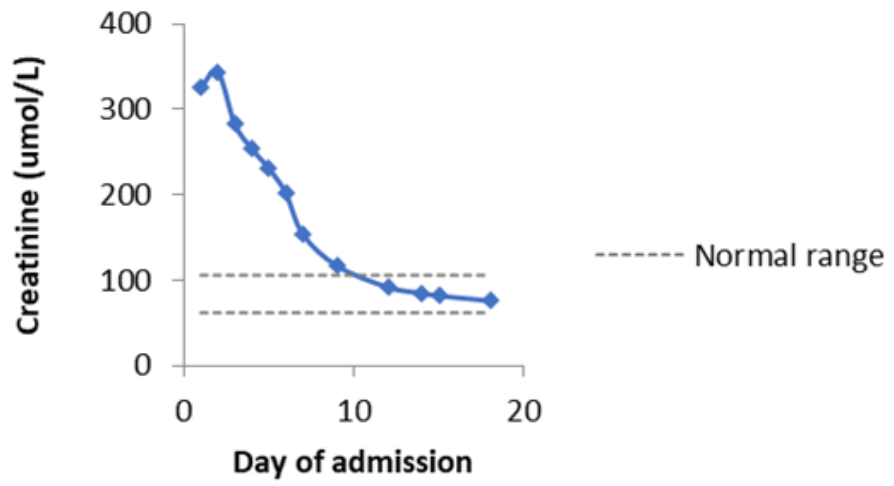

Figure I Graphs to show corrected calcium and creatinine levels during the patient's admission.

\section{Discussion}

Primary hyperparathyroidism (PHPT) is an endocrine disorder caused by overproduction of $\mathrm{PTH}$, resulting in derangement of calcium metabolism. PHPT is more common in older women, ${ }^{1}$ with $85 \%$ being asymptomatic. ${ }^{2}$ Elevated serum calcium level with raised plasma PTH level points towards a diagnosis of PHPT. However, a single normal PTH result does not exclude PHPT, as PTH is released in a biphasic circadianrhythm. ${ }^{3}$ However, incidence of n PHPT is largely unknown and possibly an early form of PHPT. Familial hypocalciuric hyercalcaemiais a rare inherited condition due to a mutation of calcium-sensing receptor gene, where patients have hypercalcaemia and hypocalciuria with normal or elevated PTH. In our case,the calcium creatinine clearance ratio $(\mathrm{Ca}: \mathrm{Cr}$ ratio) is suggestive of PHPT as it is above 0.01 , whereas in $\mathrm{FHH}, \mathrm{Ca}: \mathrm{Cr}$ ratio is usually below 0.01 . nPHPT is usually uncovered during a work up for osteoporosis where the serum calcium is normal and is renal function and vitamin $\mathrm{D}$ but PTH is elevated. Table 1 summaries the biochemical profile of the four types of htperparathyroidism.

Table I Summary of types of hyperparathyroidism and biochemistry

\begin{tabular}{lllll}
\hline Type & Serum calcium & Serum PTH & Vitamin D & $\begin{array}{l}\text { Renal } \\
\text { function }\end{array}$ \\
\hline PHPT & raised & $\begin{array}{l}\text { Raise or } \\
\text { normal }\end{array}$ & normal & normal \\
nPHPT & normal & Raise & Normal & normal \\
$\begin{array}{l}\text { Secondary } \\
\text { hyperparathyroidism }\end{array}$ & Normal or reduced & Raise & $\begin{array}{l}\text { Usually Low(look for other } \\
\text { causes e.g medication) }\end{array}$ & $\begin{array}{l}\text { Usually } \\
\text { normal }\end{array}$ \\
Tertiary hyperparathyroidism & Raised & Raised & Normal or low & abnormal \\
\hline
\end{tabular}

Our patient had nPHPT. She was receiving calcium and vitamin D supplements and was unable to attend her yearly blood test due to COVID-19. Her PTH was not checked prior to commencement of calcium and vitamin D. nPHPT was unmasked and she presented with symptomatic hypercalcaemia requiring hospitalisation. She developed a transient hypocalcaemia following hydration and bisphosphonates. This is a well described phenomenon. Her transient low serum calcium resulted in feedback to raise PTH secretion, increasing resorption of calcium from bone, absorption from the GI tract and tubular reabsorption to normalise serum calcium. ${ }^{4}$ Four months post discharge, her serum calcium remains normal and PTH remains elevated, further supporting a diagnosis of normocalcaemic PHPT.

The older person is more likely to be on calcium and vitamin D supplements, often initiated after assessment of falls. Often than not, PTH assay is not requested. For patients with PHPT and $\mathrm{nP}$ TPH who are osteoporotic and at risk of falls, they should receive vitamin D supplementation without calcium. As a safe guard, serum calcium should be monitored before and after initiation of calcium and vitamin D supplementation in the older person.

\section{Declaration of consent}

Written consent for publication of clinical details was obtained from patient.

\section{Funding}

None.

\section{Availability of data and material}

Not applicable.

\section{Code availability}

Not applicable.

\section{Acknowledgments}

None.

\section{Conflicts of interest}

The authors declare that there is no conflict of interest.

\section{References}

1. Yeh MW, Ituarte $\mathrm{PH}, \mathrm{Zhou} \mathrm{HC}$, et al. Incidence and prevalence of primary hyperparathyroidism in a racially mixed population. J Clin Endo crinol Metab. 2013;98(3):1122-1129.

2. Khan AA, Hanley DA, Rizzoli R, et al. Primary hyperparathyroidism: review and recommendations on evaluation, diagnosis, and management. A Canadian and international consensus. Osteoporos Int. 2017;28(1):119. 
3. Ghada EF, Elizabeth BK, Emery NB, et al. The parathyroid hormone circadian rhythm is truly endogenous-a general clinical research center study. J Clin Endo crinol Metab. 1997;82(1):2810286.
4. Chestnut CH, Harris ST. Short-term effect of alendronate on bone mass and bone remodeling in postmenopausal women. OsteoporoInt. 1993; 3:S17-19. 